\title{
Gestion des déchets dangereux et responsabilité sociale des firmes : le commerce illégal de déchets électriques et électroniques
}

\section{Faouzi Bensebaa et Fabienne Boudier}

\section{(2) OpenEdition}

\section{Édition électronique}

URL : http://journals.openedition.org/developpementdurable/4823

DOI : 10.4000/developpementdurable.4823

ISSN : 1772-9971

Éditeur

Association DD\&T

\section{Référence électronique}

Faouzi Bensebaa et Fabienne Boudier, « Gestion des déchets dangereux et responsabilité sociale des firmes : le commerce illégal de déchets électriques et électroniques », Développement durable et territoires [En ligne], Varia (2004-2010), mis en ligne le 29 juillet 2014, consulté le 19 avril 2019. URL: http://journals.openedition.org/developpementdurable/4823 ; DOI : 10.4000/ developpementdurable.4823

Ce document a été généré automatiquement le 19 avril 2019



Développement Durable et Territoires est mis à disposition selon les termes de la licence Creative Commons Attribution - Pas d'Utilisation Commerciale 4.0 International. 


\title{
Gestion des déchets dangereux et responsabilité sociale des firmes : le commerce illégal de déchets électriques et électroniques
}

\author{
Faouzi Bensebaa et Fabienne Boudier
}

1 En mars 2007 était lancée officiellement l'initiative mondiale du Programme des NationsUnies pour l'Environnement (PNUE) Solving the e-waste problem (StEP). L'objectif de cette initiative qui a vu le jour en raison de la dangerosité des déchets d'équipements électriques et électroniques (DEEE) ou e-déchets, est de réduire la pollution de l'environnement que provoque l'élimination ou le recyclage de ces déchets en augmentant la durée de vie des appareils et en améliorant la valorisation des matériaux qu'ils contiennent.

2 Ce problème de la gestion des DEEE s'inscrit dans la problématique plus large de la gestion des déchets dangereux dont ils représentent une part croissante. Rien que dans l'Union européenne, les déchets dangereux atteignaient 40 millions de tonnes en 2000, en augmentation de $10 \%$ par rapport à 1997 (Commission Européenne, 2006). En outre, ces déchets dangereux et plus particulièrement les DEEE, font l'objet d'un commerce international illégal. Selon l'Oclaesp ${ }^{1}, 10 \%$ du fret maritime mondial est composé de déchets dangereux et interdits à l'exportation.

3 Comment expliquer le développement du trafic de déchets dangereux, alors que d'importants dispositifs légaux ont vu le jour (notamment Convention de Bâle en 1996 et plusieurs directives européennes) et que les comportements des firmes font d'objet de l'attention soutenue tant de la communauté académique que des praticiens et des dirigeants politiques (Margolis et Walsh, 2003 ; Walsh et al., 2003) ?

Définir les comportements estimés socialement responsables (CSR) est loin d'être une tâche aisée (Maignan et Ralson, 2002 ; Roberts, 2003). Deux positions majeures peuvent être mises en évidence. La première repose sur l'adoption de critères plus ou moins objectifs à partir desquels les firmes peuvent être évaluées. Ainsi, les comportements 
socialement responsables sont ceux qui sont liés à l'octroi de salaires décents, eu égard au coût local de la vie tel qu'il est déterminé par les Nations Unies, par exemple. De même, les comportements sont socialement responsables si l'environnement local n'est pas affecté par l'entreprise, en tenant compte des standards internationalement acceptés en matière de pollution, de qualité de l'air, etc. La seconde position utilise des critères plus subjectifs et s'appuie sur la perspective dite des parties prenantes qui interagissent avec les firmes. Dans cette optique, les comportements sont socialement responsables s'ils correspondent aux attentes des parties prenantes en matière d'attitudes appropriés et acceptables.

5 L'évaluation des CSR peut varier en fonction des critères plus ou moins objectifs adoptés. En outre, ce qui est considéré comme socialement responsable évolue sur le plan historique, comme l'illustrent les changements dans le temps de la journée de travail, au moins dans les pays développés. Par ailleurs, il est nécessaire de distinguer entre la rhétorique du CSR et l'action substantive. Les entreprises peuvent en effet afficher un intérêt de pure forme dans les rapports annuels, la publicité, etc. laissant entendre qu'elles agissent de façon socialement responsable. Mais leur rhétorique peut diverger de leur comportement réel si la question n'est pas prise en compte sérieusement par l'allocation de ressources substantielles, par la mise en place d'indicateurs et de normes, etc.

Dès lors, les CSR peuvent indiquer différentes choses, à différents endroits et à différents individus. Nous devons à cet égard faire preuve de prudence dans la façon de définir et d'utiliser le concept. Tout comme nous devons être précautionneux, s'agissant de l'appréciation des CSR réels ou seulement affirmés'. Ceci est d'autant plus important que le concept est nouveau et n'est pas encore figé dans une définition universellement admise (Boxenbaum, 2006). Le choix que nous faisons dans cette recherche est que les entreprises ont des CSR par le biais de deux voies. D'une part, leurs actes ne doivent pas sciemment causer du tort à leurs parties prenantes. D'autre part, si du tort est causé, elles doivent procéder aux réparations nécessaires. Ces CSR peuvent être adoptés volontairement ou en réponse à des encouragements comme la pression morale, la pression normative, les menaces légales, les règles, les décisions des tribunaux, etc. Cette définition du CSR correspond à un comportement minimum standard au-dessous duquel les comportements deviennent irresponsables (Campbell, 2007).

7 Cette recherche se propose d'interroger les comportements des firmes du secteur des produits électriques et électroniques à même d'expliquer le développement du commerce illégal de DEEE des pays développés vers les pays en développement (PED) en mettant en tension le cadre réglementaire et le comportement des firmes. Adoptant une perspective macroéconomique, la première partie permet de caractériser les flux transfrontaliers illégaux, d'identifier les différentes réglementations en vigueur et les problèmes sanitaires et environnementaux posés. Il est à noter que les données sont particulièrement rares et manquent d'exhaustivité, tous les pays n'ayant pas ratifié la Convention de Bâle qui régit les flux transfrontaliers de déchets dangereux, ou l'ayant fait sans se sentir obligés de fournir des statistiques. La deuxième partie proposera une lecture des comportements des firmes au prisme de la perspective économique puis en utilisant les enseignements de l'analyse institutionnelle, dans la mesure où la variété de ces comportements nécessite la compréhension des différents environnements institutionnels ayant permis leur émergence. La troisième partie discutera de la 
pertinence d'impliquer les différentes parties prenantes pour assurer une meilleure efficacité des régulations.

\section{Transferts transfrontaliers de DEEE}

\subsection{Prédominance de la relation États-Unis / Chine}

Les DEEE se partagent à parts égales entre déchets électriques ( $20 \%$ de réfrigérateurs et $30 \%$ d'autres équipements électroménagers) et déchets électroniques ( $10 \%$ de téléviseurs, $25 \%$ d'ordinateurs et matériels connexes et téléphones, $15 \%$ de matériel hi$\mathrm{fi}^{3}$. Le Programme des Nations Unis pour l'Environnement (PNUE) estime leur production annuelle dans le monde à 20 à 50 millions de tonnes avec une croissance de 3 à $5 \%$ par an (PNUE, 2005), l'Agence européenne pour l'environnement à 40 millions de tonnes avec une augmentation trois fois plus rapide que les autres déchets (EEA, 2003 ; EMPA, 2007). $\mathrm{Au}$ sein des DEEE, ce sont les déchets électroniques qui augmentent le plus rapidement en liaison avec la croissance de la consommation. En premier lieu, cette dernière est liée à l'augmentation du taux d'équipement. À cet égard, une grande partie de la croissance de la production de DEEE devrait dans l'avenir provenir des pays émergents, particulièrement de Chine et d'Inde en raison de leur poids démographique. D'après Greenpeace ${ }^{4}$, en 2010, sur les 710 millions de nouveaux ordinateurs dans le monde, 178 seraient en Chine et 80 millions en Inde. En second lieu, la consommation croissante d'équipements électroniques tient au raccourcissement du cycle de vie des produits. La durée de vie d'un ordinateur est ainsi passée de 6 à 2 ans entre 1997 et 2005, celle d'un mobile est de moins de 2 ans (PNUE, 2005).

9 Les principaux producteurs de DEEE sont actuellement les pays développés, au premier rang desquels les États-Unis. D'après une étude de l'agence pour la protection de l'environnement (EPA, 2007a et 2007b), les déchets électroniques atteignaient aux ÉtatsUnis entre 1,5 et 1,9 million de tonnes en 2005. En termes de poids, 15 à $20 \%$ de ces déchets sont recyclés, les 80 à $85 \%$ restants étant incinérés ou enfouis dans des décharges. D'une façon générale, bien que le recyclage des DEEE progresse dans les pays développés, la part relative des DEEE recyclés reste stable en raison du volume croissant de déchets à recycler, lui-même lié à la très forte augmentation de la consommation d'équipements électriques et électroniques.

10 Parallèlement, une grande partie des DEEE sont exportés, souvent illégalement, des pays développés vers les pays en développement. Selon Greenpeace $^{4}, 12$ millions de tonnes de DEEE seraient envoyés annuellement en Asie. En 2003, d'après le CNIID ${ }^{5}, 23000$ tonnes de déchets électroniques auraient été exportées illégalement en Asie (Chine, Inde, Pakistan) et en Afrique de l'Ouest. Le plus souvent, ces DEEE ne sont pas recyclés mais incinérés ou enfouis, transformant ainsi les PED en poubelle des pays développés. Par exemple, selon Toxics Link (2004), $70 \%$ des DEEE mis en décharge à New Delhi (Inde) proviennent de pays développés. Plus généralement, selon l'Association chinoise des appareils électroménagers ${ }^{6}, 80 \%$ des DEEE exportés sont destinés aux PED d'Asie, dont $90 \%$ à la Chine (PNUE, 2005). Cette dernière occupe ainsi le premier rang des importateurs de déchets en général, de DEEE en particulier. 
Graphique 1. La Chine, premier importateur mondial de DEEE

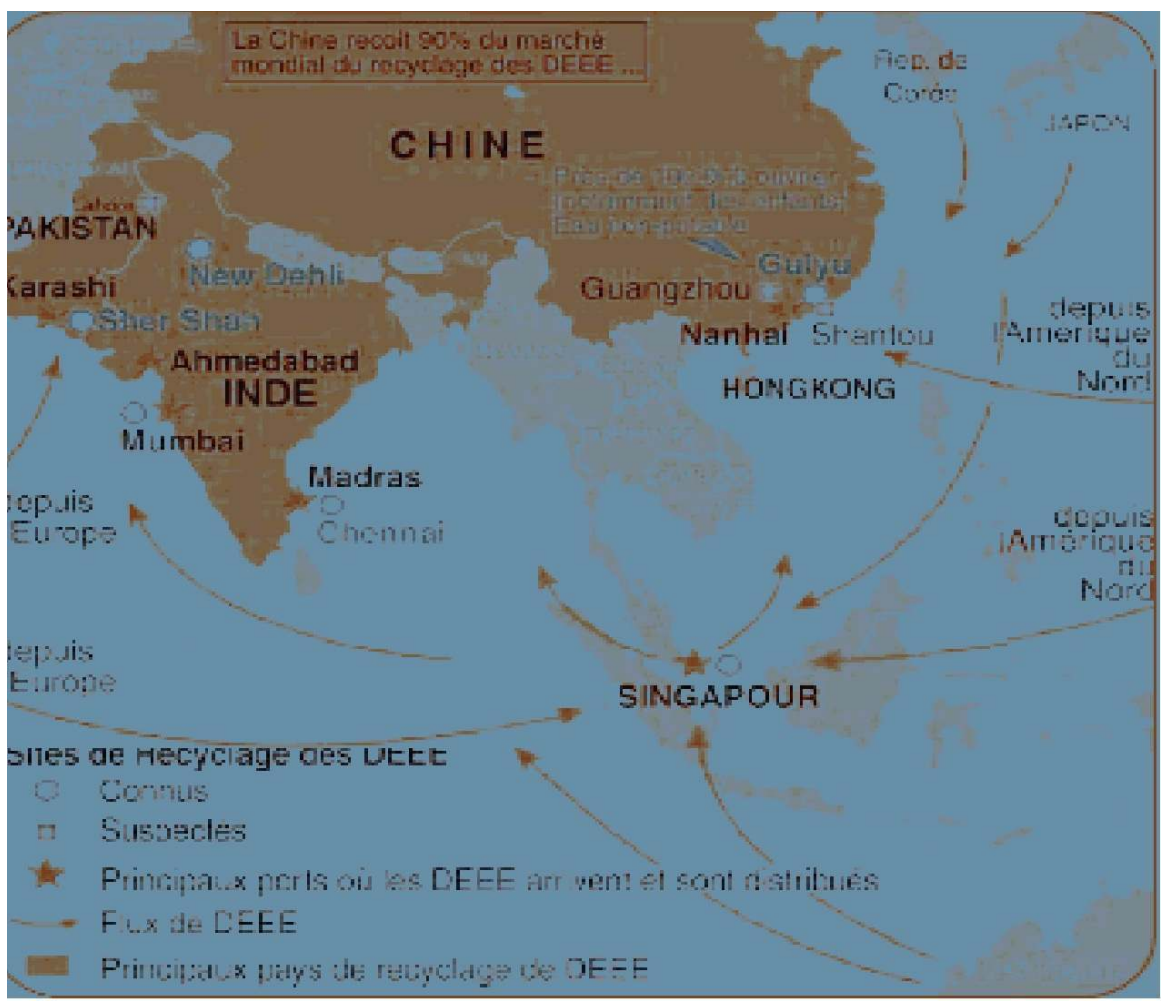

Sources : Banque Mondiale "World Development Indicators 2004", The Electronic Waste Guide, BAN, Silicon Valley Toxic Coalition, Toxic Link India, SCOPE, Greenpeace Chine 2002. in PNUE (2005), Les déchets électroniques. La face cachée de l'ascension des technologies de l'information et des communications, Bulletin d'Alerte Environnementale, Janvier.

11 La moitié des DEEE importés par la Chine provient des États-Unis, qui y envoient $80 \%$ de leurs DEEE (Puckett et Smith, 2002). Il n'existe pas de statistiques précises sur ces exportations de DEEE des États-Unis vers la Chine mais la progression des exportations de déchets en général des États-Unis vers la Chine a été telle qu'ils sont devenus le $3^{\text {ème }}$ poste d'exportation (6,1 milliards d'USD), pratiquement à égalité avec les composants électroniques (6,8 milliards) et les produits de l'aéronautique (6,3 milliards) 7 . Leur part est passée de 1,5\% en 1997 à $11 \%$ en 2006 des exportations totales des États-Unis vers la Chine 8 . En 2007, la Chine absorbait $70 \%$ des déchets exportés (officiellement) par les États-Unis. 
Graphique 2. La Chine, principal pays d'accueil des exportations de déchets des États-Unis, millions USD

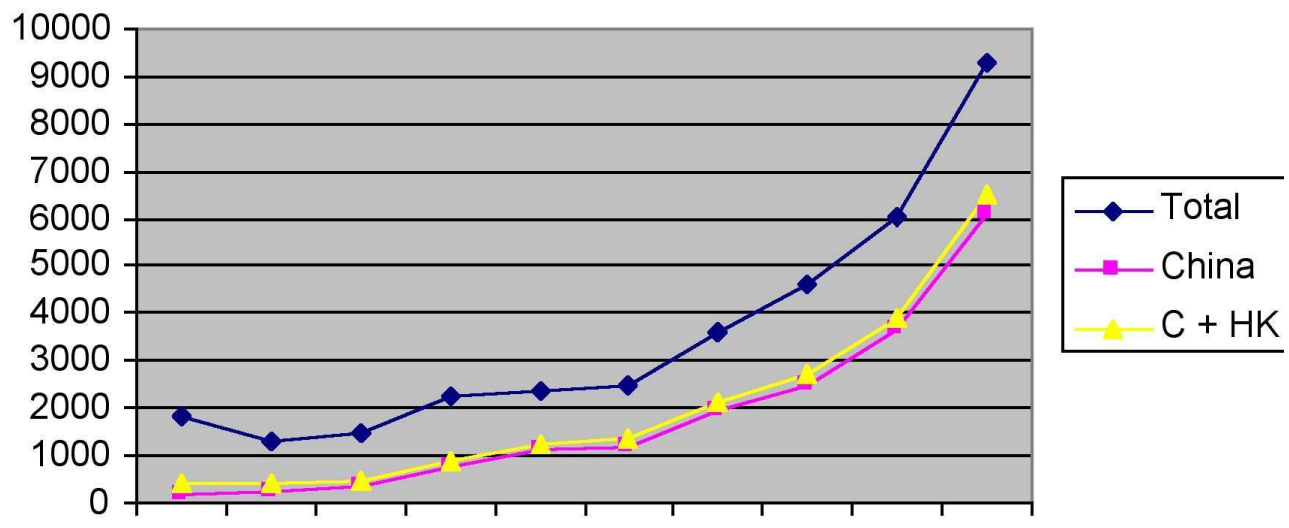

Source : Calculs des auteurs d'après les données de United States International Trade Commission.

\subsection{Les réglementations dans les pays développés}

12 A priori, ce transfert vers les PED du fardeau de la gestion des DEEE des pays développés semble paradoxal puisqu'il a lieu malgré des dispositifs juridico-réglementaires très stricts sur les mouvements transfrontaliers de déchets dangereux. Au niveau international, il n'existe pas de dispositif spécifique pour les DEEE, qui sont régis par le dispositif général de la Convention de Bâle (cf. encadré). Régionalement, le dispositif mis en place par l'Union européenne (UE) est un des plus poussés. L'UE reconnaît depuis 1993 que le principe de libre circulation des marchandises ne peut s'appliquer aux déchets. Aussi la législation européenne prévoit-elle l'interdiction de leur transfert transfrontalier en fonction de leur degré de dangerosité 9 et de la finalité de l'échange (élimination ou valorisation). Signataire du Ban Amendment de la Convention de Bâle (cf. encadré), l'UE interdit l'exportation des déchets inscrits sur la liste orange ou rouge et destinés à être valorisés et de tous les déchets destinés à être éliminés (sauf vers les pays de l'AELE parties de la Convention de Bâle). En ce qui concerne les DEEE, deux directives agissent en amont : l'une sur l'utilisation de substances toxiques dans les équipements électriques et électroniques, l'autre sur le recyclage des équipements en fin de vie.

Les réglementations européennes ont un "effet d'agenda" dans les pays membres qui doivent les mettre en application mais également hors UE, par imitation. D'une façon générale, les pays développés s'attèlent à mettre en place des filières de gestion des DEEE tout en interdisant leur exportation vers les PED. L'exception notoire est celle des ÉtatsUnis, qui n'ont pas ratifié la Convention de Bâle et où les initiatives restent du ressort des différents États. La Californie a été précurseur en la matière en adoptant une législation proche de la directive européenne DEEE, suivie en cela par l'État de Washington mais pour une entrée en vigueur en $2009^{10}$. Dans d'autres États, comme le Maine, le Minnesota et le Massachusetts, il est interdit d'éliminer les DEEE dans les décharges. Mais pour les mouvements transfrontaliers, il n'y a qu'en Californie que les exportations de DEEE sont restreintes. Outre le retard pris sur l'UE, cette absence d'une réglementation fédérale ouvre la voie à des biais et compartimente le marché. Dans les PED, l'importance du décalage accusé en termes réglementaires pose problème face à la forte croissance des déchets qu'ils ont à gérer. En effet, aux DEEE en provenance des pays développés, 
s'ajoutent de plus en plus leurs propres déchets, fruit de la consommation accrue d'équipements électriques et électroniques qu'ils connaissent. Il convient néanmoins de signaler la Convention de Bamako, adoptée sous l'égide de l'Organisation de l'Unité Africaine et qui adapte aux pays africains la Convention de Bâle (cf. encadré). Quant à la Chine, ce n'est que récemment qu'elle a mis en place un cadre législatif contraignant (cf. encadré). Elle a par ailleurs annoncé la création de trois centres de lutte contre la pollution électronique relevant d'instituts de recherche électronique ${ }^{11}$.

Encadré. Principales réglementations internationales et européennes sur les mouvements transfrontaliers de déchets dangereux et sur les DEEE

Au niveau international : 1) Convention de Bâle de 1989, entrée en vigueur en 1992 : De la volonté initiale d'interdire les mouvements transfrontaliers de déchets dangereux n'a subsisté que leur contrôle (hors nucléaire). Elle a été signée par 170 pays dont 3 ne l'ont pas ratifiée (Afghanistan, Etats-Unis et Haïti). Le Ban Amendement, qui interdit toute exportation de déchets dangereux, a été adopté en 1995 mais n'est pas encore entré en vigueur, n'ayant pas été ratifié par les trois quarts des participants. 2) Décision de l'OCDE de 1992 (C(92)39/FINAL), modifiée en 2001 (C(2001)107/FINAL) : Elle concerne les déchets entre pays de l'OCDE et introduit une distinction entre déchets non dangereux (liste verte) et déchets dangereux (listes orange et rouge) pour lesquels s'applique la Convention de Bâle.

Au niveau de l'UE

Règlement sur les transferts de déchets $\mathrm{CE} / 1013 / 2006$, entré en vigueur en juillet 2007 : Il remplace et renforce le précédent règlement $\mathrm{CE} / 259 / 93 \mathrm{du} 1^{\mathrm{er}}$ février 1993 en intégrant la révision adoptée en 2001 par l'OCDE ainsi que "l'interdiction de Bâle" et en rationalisant et précisant les procédures existantes.

Deux Directives sur les DEEE : 1) Directive DEEE 2002/96/CE, fixant pour décembre 2006 un taux minimal moyen annuel de ramassage sélectif de $4 \mathrm{~kg}$ par habitant (pour les ménages). 2) Directive RoHS (Restriction of Hazardous Substances in Electrical and Electronical Equipment) (restriction sur l'usage de certaines substances dangereuses) 2002/95/CE, limitant depuis le $1^{\text {er }}$ juillet 2006 l'utilisation de 6 substances dangereuses dans les équipements électriques et électroniques: plomb, mercure, cadmium, chrome hexavalent, PBB (polybromodiphényles) et PBDE (polybromodiphényléthers). Directive amenée à évoluer avec l'interdiction d'autres substances.

Au niveau de l'Afrique: Convention de Bamako de 1991, entrée en vigueur en 1996, relative à l'interdiction des importations de déchets dangereux et au contrôle de leurs mouvements transfrontaliers en Afrique. Adaptation de la Convention de Bâle, elle a été ratifiée par 23 pays africains (Benin, Burundi, Cameroun, Côte D'ivoire, Comores, Congo, RDC, Egypte, Ethiopie, Gabon, Gambie, Libye, Mali, Mozambique, Maurice, Niger, Ouganda, Sénégal, Soudan, Tanzanie, Togo, Tunisie, Zimbabwe).

Au niveau de la Chine

Signature de la Convention de Bâle (1996).

Loi de base de 1995, entrée en vigueur en 1996, sur la prévention et le contrôle de la pollution par les déchets solides et clause provisoire de 1996 sur l'importation de déchets solides. Cette dernière est interdite à l'exception des déchets recyclables. Un amendement de 2004 (entré en vigueur en 2005) a renforcé la loi de 1995 et impose notamment le principe "Pollueur-Payeur".

Règlement sur le recyclage des déchets de 2007, visant à imposer l'enregistrement des entreprises et le respect de normes. 
ACPEIP (Administration pour le contrôle de la pollution causée par les produits d'information électronique) de 2007 : souvent dénommée "RoHS chinoise" car semblable dans son principe à la directive européenne mais avec une étendue plus large.

\subsection{Les problèmes sanitaires et environnementaux posés par le trafic illégal des DEEE}

Les transferts de DEEE des pays développés vers les PED posent un certain nombre de problèmes environnementaux et sanitaires liés au contenu en métaux de ces déchets. Ils soulèvent tout d'abord la question générale du gaspillage de matériaux précieux et/ou rares qui ne sont pas récupérés (argent, or, palladium mais aussi bismuth, indium ou ruthénium). Ensuite, la concentration de substances toxiques bioaccumulables persistantes (barium, berrylium, cadmium, chromium, mercure, plomb, etc.) pose de graves problèmes sanitaires et environnementaux. L'importance des problèmes sanitaires tient aux conditions primitives de travail des ouvriers dans les "arrière-cours" de déassemblage des DEEE dans les PED. La manipulation des DEEE sans aucune protection et sans aucun dispositif de sécurité, les fumées cancérigènes des matières incinérées (plastiques et câbles isolants principalement), l'utilisation de bassines d'acides à l'air libre pour faire fondre les microparticules de métaux précieux contenus dans les composants électroniques, etc. exposent les ouvriers à d'importants dommages physiques : problèmes respiratoires et cardiaques, dommages au cerveau, au système nerveux, aux intestins et aux reins, impact sur le système reproducteur (par exemple, avortement des femmes vivant près des décharges), etc. Ces problèmes sanitaires sont aggravés par les problèmes environnementaux que soulève cette "gestion" sauvage et primaire des DEEE: contamination des eaux (nappes phréatiques, rivières), des sols et de l'air. Plusieurs études menées à Guiyu, un des villages du sud-est de la Chine où se concentrent ces activités informelles de traitement des DEEE, indiquent des niveaux de dioxines et de furanes dans l'air largement supérieurs aux seuils définis par l'Organisation mondiale de la santé (Leung et al., 2007; Li et al., 2007). Il en résulte des concentrations sanguines en ignifugeants ${ }^{12}$ chimiques particulièrement élevées chez les personnes travaillant à ce "recyclage" (Bi et al., 2007).

15 L'exportation, le plus souvent illégale, vers les PED dissimule souvent l'externalisation par les firmes des pays développés de la gestion de leurs DEEE. Ce faisant, elles reportent sur les PED le coût et les risques associés à cette gestion. La corrélation positive sur le plan macroéconomique entre niveau des exportations de DEEE et niveau de développement du pays d'origine laisse envisager une première explication, celle des coûts comparés de la gestion des DEEE dans les pays développés et dans les PED. L'existence de mouvements transfrontaliers de DEEE malgré des cadres juridicoréglementaires de plus en plus nombreux et contraignants suggère une seconde explication d'ordre institutionnel. Deux lectures non exclusives permettent ainsi d'expliquer les comportements des firmes, c'est-à-dire au niveau micro-économique, en matière de DEEE : économique et institutionnelle. 


\section{Externalisation par les firmes des pays développés des coûts et risques associés à la gestion des DEEE : une lecture économique et institutionnelle}

\subsection{La perspective économique}

Limpératif de maximisation du profit est la cause fondamentale qui empêche les firmes d'agir de façon socialement responsable dans l'approche contractuelle de la firme. Selon cette dernière, les intérêts dans la maximisation du profit et de la valeur sont au cœur de la firme moderne et ont la primauté sur ceux de la RSE (Friedman, 1970 ; Jensen, 2002; Levitt, 1958). En appliquant cette approche aux DEEE, la gestion des déchets peut être associée au niveau de concurrence auquel font face les entreprises. Comme le secteur des produits électroniques et électriques connaît une concurrence intense, illustrée par l'affrontement par les prix, le changement du leadership, les fusions et acquisitions, la course à la taille et à l'innovation, etc., les firmes s'efforcent de préserver leur situation financière en termes de profits et de valeur pour l'actionnaire. Cela étant, la contrainte de maîtrise des coûts et d'épargne des ressources conduit les firmes à agir de façon irresponsable. L'histoire des entreprises est jalonnée d'exemples de concurrence intense poussant les entreprises à se comporter de façon irresponsable afin de survivre : défaut de sécurité du produit, exploitation du travail, tromperie des consommateurs (Vogel, 1992). Dès lors, à moins d'un développement de nouvelles pratiques collectives, il est difficile d'augmenter les coûts de façon isolée.

En ce qui concerne les DEEE, les firmes adoptent des comportements socialement irresponsables parce qu'elles estiment que leur exportation est susceptible d'améliorer leur situation financière. S'il est admis que le recyclage des équipements électriques et électroniques (EEE) est bénéfique d'un point environnemental, son coût empêche pour le moment d'en faire une stratégie généralisée (Willems et al., 2006). Le coût d'élimination ou de recyclage des DEEE apparait comme prohibitif dans les pays développés alors que les risques encourus en contrevenant à la loi ont été jusqu'à présent relativement faibles.

L'importance de ce coût s'explique par la forte croissance de la demande de gestion de DEEE liée à la forte consommation d'EEE et au cadre réglementaire de plus en plus restrictif alors que la capacité de gestion des déchets ne s'est pas développée proportionnellement. Deux éléments principaux entrent dans la formation de ce coût : le coût $\mathrm{du}$ foncier et le coût du travail. Le coût du foncier pousse à la hausse le coût d'élimination des DEEE dans les décharges. Le coût du travail influence le coût de leur recyclage, le "dé-assemblage" des divers composants étant une activité intensive en travail alors que les possibilités d'économie d'échelle restent faibles ${ }^{13}$. Actuellement, le seul facteur susceptible de faire baisser le coût de gestion des déchets est le prix des matériaux récupérés en raison de leur rareté croissante : tel est le cas de l'indium utilisé dans les écrans plats et les téléphones mobiles, du bismuth utilisé pour le brasage sans plomb ou encore du ruthénium utilisé dans les disques durs.

19 En revanche, le coût de traitement des DEEE dans les pays PED, particulièrement dans le secteur informel, est très bas, en raison de la faiblesse du coût du travail et des moindres contraintes en termes de réglementation environnementale. D'après SVTC ${ }^{14}$, le coût de recyclage d'un ordinateur est de 2 USD en Chine contre 30 USD aux États-Unis. 
L'application de la théorie des avantages comparatifs permet d'expliquer cette mobilité internationale des DEEE, souvent sous couvert d'équipements d'occasion et/ou de dons caritatifs, des pays développés vers les PED, avec en arrière-fond l'intervention des organisations criminelles comme acteur-clé de ce trafic, qui leur est très profitable. Le développement de 1 '"écomafia"15 en Italie en est l'illustration. L'ONG italienne Legambiente estime ce marché des déchets en Italie à 3 milliards d'euros. Le trafic illégal de déchets dangereux est devenu la deuxième activité des organisations criminelles après la drogue. Souvent à la frontière du légal, le trafic de déchets dangereux s'avère moins risqué comparativement à d'autres trafics, donnant naissance à ce que Ruggiero (1997) appelle "l'économie sale" (dirty economy). En Italie, des organisations mafieuses contrôlent dans certaines régions la gestion des déchets ménagers et industriels derrière une vitrine d'activités légales : réponse aux appels d'offres des administrations locales, construction et gestion d'équipements officiels de traitement des déchets, possession d'entreprises de transport, etc. (Legambiente et al., 2003).

Les considérations précédentes conduisent à se poser le problème central du statut des DEEE et plus généralement des déchets dangereux : est-ce un bien économique comme un autre? Sur le plan juridique, la Convention de Bâle et le dispositif de l'UE répondent à cette question par la négative en édictant des normes commerciales qui en interdisent les mouvements transfrontaliers. Mais sur le plan économique, la valeur potentiellement positive ou négative du déchet dangereux conduit à considérer la question sous un autre angle. En effet, dans le cas où le déchet est recyclable, il peut être éventuellement source de profit. S'il ne l'est pas, son élimination lui confère une valeur commerciale négative. Dans le premier cas se pose le dilemme entre les avantages et les inconvénients du recyclage. En termes d'avantages, en participant à la dernière phase du cycle de vie du produit, les pays d'accueil bénéficient de matières premières à un coût faible, créent des emplois et s'approprient une partie de la création de richesses. Dans les provinces côtières du sud-est de la Chine, les activités liées au traitement des DEEE contribuent à la croissance économique locale, notamment par le biais des emplois créés. Ainsi à Guiyu, le district évoqué précédemment, plus de 100000 personnes sont employées à traiter le million de tonnes de DEEE reçu annuellement (Kojima, 2005, p. 41). Quant aux inconvénients, ils concernent principalement l'impact des déchets sur l'environnement dans lequel se déroule le recyclage et sur la santé de la population. Le second cas renvoie au fait qu'une grande partie des DEEE envoyés des pays développés vers les PED sont des DEEE dont il n'y a rien à tirer, conséquence de l'hypocrisie soulignée par le BAN de la pratique consistant à envoyer sous l'intitulé de matériel dit de seconde main des DEEE inutilisables qui finiront dans une décharge à ciel ouvert (Puckett et Smith, 2002). Cela revient pour certains acteurs de la gestion des DEEE dans les pays développés, à tout simplement reporter les coûts et risques de cette gestion sur les acteurs dans les PED, qui ne disposent pas des infrastructures et des compétences en expertise nécessaires.

\subsection{La perspective institutionnelle}

21 Le dispositif institutionnel de régulation organisé par les pouvoirs publics est censé favoriser les CSR. Comme le montre la perspective historique, le rôle de la régulation est crucial. Ainsi, aux États-Unis, la dérégulation opérée dans les années 80 et 90 a favorisé l'émergence d'un environnement dans lequel les entreprises ont pu jouir d'une grande liberté et agir de façon irresponsable. Par exemple, la crise des caisses d'épargne, le scandale d'Enron, la falsification des comptes dans un certain nombre de grandes 
entreprises, etc. peuvent être attribués, pour une bonne partie à la dérégulation financière (Stiglitz, 2003). De même, en Italie, la faible régulation du marché boursier a été avancée comme explication du scandale de l'entreprise Parmalat ${ }^{16}$. La régulation par la règlementation apparaît de prime abord comme la réponse adéquate au problème des déchets dangereux.

En ce qui concerne ces derniers, théoriquement, l'application de la seule convention de Bâle semble à même de mettre fin au trafic de DEEE. En premier lieu, elle prévoit l'interdiction des mouvements transfrontaliers de déchets dangereux qui proviennent d'un État non contractant ou pour lesquels on suspecte que le traitement ne sera pas respectueux de l'environnement. En second lieu, elle défend le principe d'"autosuffisance", c'est-à-dire le traitement des déchets dangereux dans le pays où ils ont été produits. Cette obligation d'éliminer les déchets dangereux au plus près de leur lieu de production peut être considérée comme économiquement rationnelle dans la mesure où elle obéit à un principe de précaution ${ }^{17}$ (Gollier et Reich, 2003) et évite des transports sur la longue distance. Sur la base de ces deux principes, la Convention de Bâle devrait donc conduire à limiter les mouvements transfrontières des déchets dangereux. Comment expliquer alors les mouvements transfrontaliers de DEEE ? Plusieurs éléments peuvent être pris en considération.

23 L'absence de régulations, comme par exemple l'absence de lois fédérales aux États-Unis qui n'ont pas ratifié la Convention de Bâle, reflète le manque de courage politique des États pour contrôler les entreprises nationales qui considèrent que la réglementation doit être minimale et rester au stade incitatif. Elle laisse entendre également que les États ne veulent pas contraindre les firmes, de peur de les voir quitter le territoire national, avec toutes les conséquences potentielles en termes d'emplois, d'investissements et de recettes fiscales (Ohmae, 1990, 1995). L'absence de lois affaiblit ainsi la régulation, favorise l'émergence de free rider et in fine de comportements socialement irresponsables.

Au-delà de l'absence de régulations, c'est également la capacité de l'État à surveiller le comportement des firmes et à appliquer les règles lorsque cela est nécessaire qui importe. Cette "mollesse" de la régulation est plus évidente dans les pays importateurs de DEEE que dans les pays exportateurs, en l'occurrence les PED, où les capacités institutionnelles sont plus faibles. Ce qui signifie que les pays récepteurs éprouvent des difficultés à se protéger. Cette faiblesse institutionnelle peut être expliquée en partie par un climat favorisant la corruption et la sauvegarde d'intérêts économiques de court terme. Ainsi, un accord Japon/Philippines en 2006 a aboli tous les contrôles sanitaires des déchets aux Philippines. De même, en Chine, le secteur informel du recyclage des DEEE est très difficile à contrôler et pour le moment, les entreprises "officielles" (et donc de qualité) ne peuvent le concurrencer. De surcroît, la législation chinoise mise en place en 2005 semble également bien molle: elle interdit l'importation de déchets solides à l'exception des déchets recyclables, cette exception ouvrant la porte aux importations de DEEE à éliminer et requalifiés de recyclables. En même temps, les autorités laissent faire dans un souci de paix sociale et de clientélisme.

Dans cette perspective, les CSR en matière de DEEE ne peuvent émerger que s'il existe un système de régulation bien organisé et d'autant plus efficace qu'il est fondé sur la menace perçue de l'intervention de l'État et que ce dernier fournit un support pour cette forme de gouvernance. Les firmes ne se plieront à une gestion des déchets dangereux compatible avec l'environnement que si la loi les y contraint, en d'autres termes que si les fabricants sont tenus juridiquement responsables des effets secondaires sur le public. Des auteurs 
(Vogel, 1989 par exemple) estiment que les firmes peuvent non seulement résister aux régulations mais peuvent également chercher à les contrôler dans un sens qui leur est favorable. Dès lors, un certain nombre d'éléments dépendent des dispositifs institutionnels et de la configuration de la régulation ainsi que de l'équilibre des forces politiques les sous-tendant (Campbell, 1988 ; Vogel, 1986).

En matière de DEEE, les firmes font preuve d'un fort lobbying auprès de la Convention de Bâle et de l'UE. Ainsi, en Suisse, les industriels ont réussi à empêcher la classification des téléphones portables parmi les produits toxiques. De même, en 2000, l'American Electronics Association a menacé de poursuivre l'UE devant l'Organisation mondiale du commerce pour entrave au libre-échange.

plus, les dispositifs institutionnels peuvent ne pas être appliqués pour des raisons diverses : manque de moyens financiers et/ou humains, insuffisance de la formation du personnel, pléthore des documents à fournir, etc. Ceci conduit à la dissimulation et à la difficulté de désigner les autorités compétentes. In fine se pose la question de savoir si l'inflation réglementaire n'est pas contraire à la recherche de l'efficacité. Le fait que le développement du trafic international de déchets dangereux à partir des années 80 a coïncidé avec le développement de normes environnementales appuierait cette hypothèse. Trop de législation, en augmentant le coût du traitement des DEEE, peut conduire à des éliminations illégales et par ricochet réduire les revenus de l'industrie de la gestion des déchets. La persistance des flux de transfert des déchets dangereux, voire leur croissance, souligne l'imperfection des dispositifs institutionnels. Se pose dès lors le problème de l'implication des parties prenantes.

\section{Implication des parties prenantes}

En matière de DEEE, les parties prenantes suivantes retiennent l'attention : les États (non pas en tant qu'abstraction mais via notamment les organes de surveillance, de contrôle et de sanction), les collectivités locales, les "fabricants" d'EEE, les distributeurs, les organismes de transport, les consommateurs, les ONG qui font pression sur les producteurs pour traiter le problème en amont (principe de l'écoproduction) et en aval (récupération et traitement des déchets). Le rôle central des collectivités locales est à souligner dans la mesure où elles ont la charge des déchets des ménages où se retrouve la plupart des DEEE. ${ }^{18}$

Les CSR relèvent en partie de la capacité des acteurs externes (notamment syndicats, consommateurs, $\mathrm{ONG}$ ) à participer aux processus de régulation et à les surveiller (Troast et al., 2002). Les dispositifs institutionnels en matière de DEEE se veulent surtout coercitifs, alors que les États sont plus efficaces dans la facilitation du CSR s'ils permettent par exemple à tous les acteurs concernés de participer à l'élaboration des textes, à l'accès à l'information (pour les consommateurs par exemple), etc. Les firmes auront tendance à agir de façon socialement responsable si le processus par lequel ces régulations et ces capacités d'application sont développées est fondé sur le consensus et la négociation entre elles, les pouvoirs publics et les autres parties prenantes. Par exemple, la pollution de l'air a été règlementée aux États-Unis et en Suède à la fin des années 60 et au début des années 70 mais de façon différente dans chacun des pays et avec des résultats différents. En Suède, le processus s'est appuyé sur la consultation des entreprises, des spécialistes de l'environnement, des scientifiques, des agences publiques et des partis politiques. Ceci a permis d'aboutir à un ensemble de régulations 
correspondant aux technologies disponibles et tenant compte tant des conséquences économiques qu'environnementales. Les firmes et les autres parties impliquées dans la négociation étaient satisfaites et la mise en œuvre a été efficace. La forte culture du consensus social, en usage en Suède, a certainement dû jouer un rôle dans ce succès ( Kelman, 1981; Lubatkin et al., 2005 ; Lunqvist, 1980). Aux États-Unis, le processus a été plus conflictuel, les régulations adoptées se sont révélées ardues à mettre en œuvre, les standards mis en place étant difficiles à atteindre à cause des technologies disponibles. Dès lors, les firmes ont lutté contre l'introduction des régulations qui, en fin de compte, furent moins efficaces qu'en Suède (Lundqvist, 1980 ; Vogel, 1986).

Partant de là, l'implication des parties prenantes en matière de DEEE passe par l'application du principe du pollueur-payeur ("pay-as-you-throw" principle), dispositif contraignant obligeant les firmes à supporter le coût environnemental supporté jusqu'alors par la collectivité ${ }^{19}$. Initialement, ce principe reposait sur un dépôt de garantie obligatoire lors de la mise sur le marché d'un EEE. L'application du principe permet en théorie de savoir qui doit payer mais en fait, il s'avère difficile à appliquer aux produits qui deviennent des déchets en fin de vie, c'est-à-dire dans le cadre d'une logistique inversée ou verte. A priori, sur la base des seuls droits de propriété, le consommateur apparait comme seul responsable et d'un point de vue opérationnel, faire peser le coût du recyclage sur lui semble la solution la plus facile. Pourtant, il n'a qu'un faible pouvoir d'influence sur la composition du produit. La responsabilité du producteur se trouve donc également questionnée, en particulier quant aux problèmes posés par l'élimination des substances dangereuses contenues dans les EEE et par le dé-assemblage des équipements en fin de vie. Dès lors, il apparaît plus pertinent d'adopter le principe de responsabilité élargie du producteur (REP), en considérant ce dernier comme responsable de tout le cycle de vie du produit par l'obligation de reprendre le produit en fin de vie et de le faire traiter. Cette REP constitue une incitation à internaliser la gestion des DEEE et à la faire apparaitre dans le prix et dès lors, à aller vers une éco-conception, pour diminuer le coût du recyclage et donc agir sur la compétitivité des producteurs. Reste néanmoins posé le problème des comportements de free rider, une firme pouvant par exemple disparaître en laissant les autres assumer la gestion des DEEE issus de ses activités.

Dans une approche par les parties prenantes (au sens de Freeman, 1984 et de Mitchell et al., 1997), la directive de l'UE sur les DEE implique différents acteurs tout en concernant surtout les municipalités. Cette directive a fixé le taux minimal moyen annuel de ramassage sélectif à $4 \mathrm{~kg}$ par habitant à partir de décembre 2006 (pour les ménages). Par ailleurs, elle oblige les distributeurs à proposer la reprise gratuite de l'ancien appareil lors de la vente d'un nouvel appareil similaire. Elle contraint le consommateur à payer une écotaxe et à faire le tri des ordures ménagères. Les États membres de l'Union européenne ont pour leur part obligation de s'assurer que les déchets sont identifiables et de prendre des mesures pour que les consommateurs ne jettent plus les DEEE avec les ordures ménagères. Quant au producteur, la directive lui impose le financement de la collecte des DEEE des ménages, du traitement, de la valorisation et de l'élimination des DEEE à compter du 15/08/05. Il peut s'acquitter de cette obligation soit par un système individuel ayant reçu l'agrément des pouvoirs publics ou par un système collectif en adhérant à un éco-organisme. Plus en amont, le producteur doit éliminer dans les produits eux-mêmes six substances dangereuses. Le nouveau cadre réglementaire induit pour les firmes chargées de la collecte et/ou du traitement des adaptations au nouveau cadre réglementaire en termes d'organisation et d'investissement. Les collectivités 
locales se doivent d'aménager les déchetteries à la collecte sélective des DEEE. Les écoorganismes, outre leur rôle d'information et de sensibilisation du public, assurent la logistique nécessaire à l'enlèvement des DEEE et contrôle leur recyclage.

Pour l'essentiel, l'implication des parties prenantes se fait par le haut, c'est-à-dire de façon coercitive, alors que la régulation peut s'affranchir de l'État, notamment au niveau sectoriel. En effet, souvent, les acteurs d'un secteur donné peuvent établir leurs propres mécanismes de régulation par la mise en place de standards pour assurer des pratiques responsables, particulièrement en termes de qualité du produit, de sécurité du travail, etc. De fait, le moyen le plus efficace pour obtenir des comportements socialement responsables passe par la pression exercée sur les "pairs" du secteur. Cette pression peut être le fait des associations industrielles dont le souci est d'obtenir de leurs membres un CSR. Dès lors, la capacité des parties prenantes à surveiller la régulation sectorielle contribue à déterminer politiquement l'efficacité de la régulation et augmente la probabilité que les firmes se comportent de façon responsable, surtout quand elles opèrent dans un espace international.

Toutefois, cette démarche ne semble pas présente en matière de DEEE. En particulier, le recyclage est plus perçu par les producteurs d'EEE comme un fardeau que comme une source d'opportunités commerciales, comme le met en évidence l'étude réalisée par l'IPTS (2006) pour la Commission européenne. La pression peut également venir des acteurs collectant les déchets et/ou les valorisant. La collaboration entre les trois types d'acteurs est à même de réduire l'impact environnemental des déchets. Enfin, la pression sur les acteurs peut passer par l'émergence d'organisations indépendantes (ONG, mouvements sociaux, investisseurs, et presse) qui établissent des codes afin de surveiller les acteurs et les pousser à modifier leurs comportements, par le développement d'un environnement institutionnalisé (écoles, publications, etc.), par le dialogue avec différents acteurs sociaux (syndicats, employés, groupes de communauté, investisseurs et autres parties prenantes). Certaines ONG dénoncent le commerce illégal de déchets toxiques en général (le Basel Action Network ou BAN, par exemple) ou plus généralement, luttent en faveur d'une réduction des déchets à la source ( $c f$. la Coordination pour la réduction des déchets à la source en France). Paradoxalement, les citoyens dans le cadre de la protection de leur environnement peuvent être amenés à contester l'installation d'une décharge ou d'une usine d'incinération de déchets sans se préoccuper des alternatives, dont l'exportation illégale vers le PED. A ces comportements Nimby (Not In My Back Yard), soucieux uniquement d'un intérêt particulier, s'opposent les mouvements Nope (Not On Planet Earth).

34 À vrai dire se pose ici le problème de l'information. La question des déchets dangereux semble souvent méconnue et les firmes font preuve d'asymétrie d'information, notamment à l'égard des consommateurs en matière de dangerosité des produits vendus. La perception de cette dangerosité par les consommateurs en particulier et plus généralement par les citoyens demeure relativement faible. En matière de DEEE, si le trafic est unanimement reconnu dans les pays développés, il reste essentiellement posé comme un problème entre États sans prise en considération et implication véritable de toutes les parties prenantes, notamment des agents économiques concernés dans les pays récepteurs des déchets. 


\section{Conclusion}

35 Cette recherche s'est intéressée aux comportements des acteurs du secteur des produits électriques et électroniques et s'est attachée à analyser les raisons du développement de comportements socialement irresponsables via les transferts transfrontaliers de DEEE. Ceux-ci s'avèrent motivés par des considérations économiques ou institutionnelles, les deux pouvant être interdépendantes. Dans un contexte de mondialisation croissante, les acteurs des pays développés peuvent en effet reporter le coût et les risques associés à l'élimination des DEEE sur les PED dans lesquels les coûts de traitement sont plus faibles et le cadre institutionnel plus "mou". Paradoxalement ils y sont incités dans les deux cas extrêmes d'absence de régulation (États-Unis) ou de fortes contraintes institutionnelles (UE). Dans le dernier cas, le "surcoût" entraîné par le respect des dispositifs réglementaires peut expliquer des comportements socialement irresponsables mais ceuxci peuvent être également générés, comme dans le premier cas, par une faiblesse de la gouvernance, la mollesse de la régulation conduisant aux mêmes types de comportements socialement irresponsables que l'absence de régulation. Ce constat nous a conduit à nous interroger sur les voies à suivre pour faire émerger des CSR. Plus particulièrement, il amène à se demander si au-delà de la voie coercitive à travers l'interdiction des mouvements transfrontaliers de DEEE, la solution ne passe pas par l'implication des parties prenantes, à travers la mise en place généralisée de codes de conduite et/ou les pressions exercées sur les acteurs.

Cette recherche laisse un certain nombre de questions ouvertes. Tout d'abord, l'interdiction des mouvements transfrontaliers de DEEE basée sur le principe d'autosuffisance de la Convention de Bâle, peut se révéler inefficace en entraînant une élimination non optimale des déchets dans le pays d'origine si ce dernier n'en possède pas la capacité technique par exemple. En d'autres termes, la question de la supériorité d'un traitement adéquat avec mouvement transfrontalier par rapport à un traitement sur place se doit d'être posée même si pour le moment, elle n'est pas vraiment d'actualité dans la mesure où les flux de DEEE vont des pays développés vers les PED. Cela étant, se pose le problème des transferts de technologie des premiers vers les derniers. Outre que ces transferts de technologie pourraient permettre aux PED de mieux gérer les DEEE accueillis et/ou produits localement, ils pourraient être envisagés dans le cas de transferts transfrontaliers comme une contrepartie de l'exportation de risques et de coûts associés à la gestion des DEEE par les pays développés.

37 Ensuite dans la délimitation des responsabilités en matière de DEEE, se pose la question de l'identification du producteur. Est-ce celui qui usine les produits, c'est-à-dire le manufacturier, auquel cas l'exportation des DEEE vers l'Asie et particulièrement vers la Chine pourrait être considérée comme un juste retour. Ou bien, est-ce celui qui contrôle et qui est donneur d'ordres? Les précautions terminologiques semblent ici importantes.

Enfin, outre les déchets en provenance des pays développés, les PED vont devoir faire face à la croissance de leurs propres DEEE en liaison avec l'évolution rapide de leur demande domestique d'EEE. À la lumière des leçons tirées de la gestion des DEEE par les pays développés, il apparaît que les PED doivent mettre en place des dispositifs institutionnels lorsque ceux-ci font défaut ou s'assurer de leur bonne application. En tout état de cause, dans les deux cas de figure, l'ouverture des dispositifs aux différentes parties prenantes semble incontournable. 


\section{BIBLIOGRAPHIE}

Bi X., Thomas G. O., Jones K.C., Qu W., Sheng G., Martin F.L., Fu J. (2007), Exposure of Electronics Dismantling Workers to Polybrominated Diphenyl Ethers, Polychlorinated Biphenyls, and Organochlorine Pesticides in South China, Environmental Science \& Technology., 41(16), 5647 -5653.

Boxenbaum E. (2006), Corporate Social Responsibility as Institutional Hybrids, Journal of Business Strategies, 23(1), 45-64.

Campbell J. L. (1988), Collapse of an Industry: Nuclear Power and the Contradictions of U.S. Policy, Ithaca, New York : Cornell University Press.

Campbell J. L. (2007), Why Would Corporations Behave in Socially Responsible Ways? An Institutional Theory of Corporate Social responsibility, Academy of Management Review, 32(3), 946-967.

Clarkson M. B. (1995), A Stakeholder Framework for Analyzing and Evaluating Corporate Social Performance, Academy of Management Review, 20 (1), 92-117.

Coase R. H. (1960), The Problem of Social Sost, The Journal of Law and Economics, 3 (October), 1-44.

Commission Européenne (2006), Production, traitement et transferts transfrontaliers de déchets dangereux et autres déchets dans les Etats membres de l'Union européenne (1997-2000), Rapport de la Commission au Conseil et au Parlement Européen, téléchargeable sur le site eur-lex.europa.eu.

EEA (European Environmental Agency) (2003), Waste from Electrical and Electronic Equipment (WEEE) - Quantities, Dangerous Substances and Treatment Methods, January, www.eea.europa.eu

EMPA (2007), Une initiative de l'ONU en faveur de l'amélioration du recyclage des déchets électroniques, Communiqué aux médias, 7 mars.

EPA (Environmental Protection Agency) (2007a), Management of Electronic Waste in the United States, Approach one, Draft final report, EPA530-R-07-004a, Avril, www.epa.gov/e-Cycling/docs/app-1.pdf

EPA (Environmental Protection Agency) (2007b), Management of Electronic Waste in the United States, Approach two, Draft final report, EPA530-R-07-004b, Avril, www.epa.gov/e-Cycling/docs/ app-1.pdf.

Freeman R. E. (1984), Strategic Management : A Stakeholder Approach, Pitman.

Friedman M. (1970), The Social Responsibility of Business is to Increase Profits, New York Times Magazine, September 13, 32-33.

Gollier C., Reich N. (2003), Decision-making under Scientific Uncertainty : The Economies of the Precautionary Principle, The Journal of Risk and Uncertainty, 27(1), 77-103.

IPTS (Institute for Prospective Technological Studies) (2006), Implementation of Waste Electric and Electronic Equipment Directive in EU 25, étude réalisée pour la Commission européenne.

Jensen M. (2002), Value Maximization, Stakeholder Theory, and the Corporate Objective Function, Business Ethics Quarterly, 12, 235-256. 
Kelman S. (1981), Regulating America, regulating Sweden : A comparative study of occupational safety and health policy. Cambridge : Massachusetts Institute of Technology Press.

Kojima M. (ed.) (2005), International Trade of RecyclableResources in Asia, Institute of Developing Economies.

Kourilsky P. et Viney G. (2000), Le Principe de Précaution, Paris : La Documentation Française.

Legambiente, Gruppo Abele-Nomos et GEPEC-EC (2003), The Illegal Trafficking in hazardous waste in Italy and Spain, Final report.

Leung A. O.W., Luksemburg W.J., Wong A.S. et Wong M.H. (2007), Spatial Distribution of Polybrominated Diphenyl Ethers and Polychlorinated Dibenzo-P-Dioxins and Dibenzofurans In Soil and Combusted Residue at Guyiu, an Electronic Waste Recycling Site in Southeast China, Environmental Science \& Technology, 41(8), 2730-2737.

Levitt T. (1958), The Dangers of Social Responsibility, Harvard Business Review, 36(5), 41-50.

Li H., Yu L., Sheng G., Fu J., Peng P. (2007), Severe PCDD/F and PBDD/F Pollution in Air around an Electronic Waste Dismantling Area in China, Environmental Science \& Technology, 41(16), 5641 $-5646$.

Lubatkin M., Lane P.J., Collin S.O., Very P. (2005), Origins of Corporate Governance in the USA, Sweden and France, Organizational Studies, 26(6), 867-888.

Lundqvist L. (1980), The Hare and the Tortoise : Clean Air Policies in the United States and Sweden, Ann Arbor : University of Michigan Press.

Maignan I., Ralson D.A. (2002), Corporate Social Responsibility in Europe and The U.S. : Insights from Businesses Self-Presentations, Journal of International Business Studies, 33, 497-514.

Margolis J. D., Walsh J. P. (2003), Misery Loves Companies : Rethinking Social Initiatives by Business, Administrative Science Quarterly, 48, 268-305.

Mitchell R. K., Agle B. R. et Wood D. J. (1997), Toward a Theory of Stakeholder Identification and Salience : Defining the Principle of Who and What Really Counts, Academy of Management Review, 22(4), 853-886.

Ohmae K. (1990), The Bordeless World: Power and Strategy in the Interlinked Economy, New York : Harper Collins.

Ohmae (1995), The End of the Nation State, New York : Free Press.

Pigou A. C., (1920), The Economics of Welfare, London : Macmillan.

PNUE (Programme des Nations Unies pour l'Environnement) (2005), Les déchets électroniques. La face cachée de l'ascension des technologies de l'information et des communications, Bulletin d'Alerte Environnementale, Janvier.

Puckett J. et Smith T. (2002), Exporting Harm : The High-Tech Trashing of Asia, The Basel Action Network and Silicon Valley Toxics Coalition, www.ban.org/E-waste/technotrashfinalcomp.pdf. Roberts (2003), The Manufacture of Corporate Social Responsibility : Constructing Corporate Sensibility, Organization, 10, 249-265.

Ruggiero V. (1997), Criminals and Service-Providers : Cross-national Dirty Economies, Crime, Law and Social Change, 28.

Sénat (2002), Projet de loi autorisant l'approbation de l'amendement à la convention de Bâle sur le contrôle des mouvements transfrontaliers de déchets dangereux et leur élimination, rapport $\mathrm{n}$ - 343 (2001-2002) de Robert del Picchia. 
Sodhi M. S. et Reimer B. (2001), Models for Recycling Electronics End-of-Life Products, $O R$ Spektrum, 23, 97-115.

Stiglitz J. E. (2003), The Roaring Nineties : A New History of the World's Most Prosperous Decade, New York : Norton.

Toxics Link (2004), E-waste in Chennai : Time is Running out, www.toxicslink, consulté en septembre 2007.

Troast J. G., Hoffman A. J., Riley H. C., Bazerman M. H. (2002), Institutions as Barriers and Enablers to Negotiated Agreements : Institutional Entrepreneurship and the Plum Creek Habitat Conservation Plan, in A. J. Hoffman, M. J. Ventresca (Eds.), Organizations, Policy, and the Natural Environment, Stanford, CA : Stanford Unversity Press, 235-261.

Vogel D. (1986), National Styles of Regulation: Environmental Policy in Great Britain and the United States, Ithaca, New York : Cornell University Press.

Vogel D. (1989), Fluctuating Fortunes : The Political Power of Business in America, New York : Basic Books.

Vogel D. (1992), The Globalisation of Business Ethics : Why America Remains Distinctive, California Management Review, 35(1), 30-49.

Walsh J.P., Weber K. et Margolis J. D. (2003), Social Issues and Management : Our Lost Cause Found, Journal of Management, 29, 859-881.

Wayne M. (2007), China-U.S. trade issues, CRS Report for Congress, 3 mai.

Willems B., Dewulf W et Duflou J.R. (2006), Can Large-Scale Disassembly be Profitable? A Linear Programming Approach to Quantifying the Turning Point to Make Disassembly Economically Viable, International Journal of Production Research, 44(6), 1125 - 1146

\section{Sources secondaires utilisées}

BAN (Basel Action Network), réseau d'action pour l'application de la convention de Bâle, www.ban.org.

CHEAA (Association chinoise des appareils électroménagers), http://www.cheaa.org

CNIID (Centre national d'information indépendante sur les déchets), http://www.cniid.org

EMPA (Institut de recherche suisse en science des matériaux et en technologie), www.empa.ch.

Legambiente, association environnementale italienne à l'origine du concept d'écomafia, http:// www.legambiente.com

NOVETHIC, media en ligne du développement durable, http://www.novethic.fr

OCLAESP (Office Central de Lutte Contre les Atteintes à l'Environnement et à la Santé Publique), organisme rattaché à la gendarmerie nationale et non compétent pour les stupéfiants, armes, munitions, produits explosifs et matériels nucléaires, http://www.defense.gouv.fr/gendarmerie/ decouverte/missions/police_judiciaire/offices_centraux/oclaesp

SEPA (State Environmental Protection Administration of China, Agence chinoise de protection de l'environnement), http://www.sepa.gov.cn .

SVTC (Silicon valley toxics coalition), http://www.svtc.org

Toxics link, association environnementale indienne, http://www.toxicslink.org

PNUE (Programme des Nations Unies pour l'Environnement), www.unep.org 


\section{NOTES}

1. Office Central de Lutte Contre les Atteintes à l'Environnement et à la Santé Publique.

2. Ces CSR sont issus eux-mêmes du concept plus englobant de responsabilité sociale de l'entreprise (RSE).

3. Basel Action Network, Sodhi et Reimer (2001) in PNUE (2005).

4. What is e-waste?, www.greenpeace.org/usa/campaigns/toxics/hi-tech-highly-toxic/e-waste .

5. Fiche synthèse DEEE, www.cniid.org, consulté en septembre 2007.

6. CHEAA, www.cheaa.com, consulté en septembre 2007.

7. Chiffres issus de Wayne (2007).

8. Calculs des auteurs d'après les données de United States International Trade Commission.

9. Toxique, explosif, corrosif, inflammable, comburant, infectieux, écotoxique (Sénat, 2002).

10. Législation des DEEE : Progrès au Nord, lacunes au Sud, Dossier Novethic, 31 mai 2006, www.novethic.fr.

11. La Chine s'attaque aux appareils électroniques, Journal de l'Environnement, 17 juillet 2007, www.journaldelenvironnement.net.

12. Produits rendant ininflammables des objets combustibles.

13. Ainsi, on constate que plus un pays est petit, plus le trafic de déchets dangereux est important.

14. Silicon Valley Toxics Coalition, www.svtc.org, consulté en septembre 2007.

15. Terme introduit en 1994 par Legambiente, association environnementale italienne.

16. The Economist, The crisis at Parmalat, 370, 3 janvier 2004, 45-46.

17. D'une manière précise, l'article premier de la loi Barnier du 2 février 1995 en France énonce que le principe de précaution est le principe selon lequel "l'absence de certitude, compte tenu des connaissances scientifiques et techniques du moment, ne doit pas retarder l'adoption de mesures effectives et proportionnées visant à prévenir un risque de dommages graves et irréversibles à un coût économiques acceptable" (Kourilsky et Viney, 2000).

18. Trois approches permettent de définir les parties prenantes. La première, celle de Freeman (1984), considère qu'une partie prenante porte sur tout groupe ou individu qui peut affecter ou être affecté par la mise en œuvre des objectifs de l'organisation. La deuxième, celle de Mitchell et al. (1997), met l'accent sur les parties prenantes à considérer, c'est-à-dire celles saillantes et légitimes. La troisième, celle de Clarckson (1997), distingue les parties prenantes primaires de celles qui sont secondaires. Les premières font l'objet de davantage d'attention de la part des entreprises.

19. Ce principe a été développé par Pigou (1920) sur la base de la distinction entre produit marginal privé et produit marginal social. Selon Pigou, l'existence d'externalités justifie l'intervention de l'Etat pour qu'elles soient prises en compte dans le calcul économique. Il préconise ainsi de taxer les activités à l'origine d'externalités négatives et inversement de subventionner les activités à l'origine d'externalités positives. A l'inverse, selon Coase (1950), l'intervention de l'État n'est pas nécessaire. Sur la base de droits de propriété bien définis, l'internalisation des externalités se fait via la négociation marchande entre pollueurs et pollués. 


\section{RÉSUMÉS}

Prenant les déchets électriques et électroniques comme terrain d'observation, cette recherche étudie les fondements du commerce illégal de déchets dangereux des pays développés vers les PED. En effet, malgré la multiplication des législations, le problème des déchets dans les pays développés, loin d'être traité en amont, l'est en grande partie en aval par leur délocalisation dans les PED. Les deux lectures proposées, économique et institutionnelle, permettent de montrer comment les contraintes de coût de gestion des déchets dans les pays développés conjuguées à l'imperfection et à la non-application des législations conduisent à des comportements socialement irresponsables. À partir de là, l'implication des parties prenantes est suggérée comme solution pour éliminer ce type de comportements.

Based on the analysis of the waste electrical and electronic equipments (WEEE), the research investigates the motivations for the illegal cross-border trade in hazardous wastes from developed countries to developing countries. Although many developed countries have implemented waste shipment regulations, they are passing the risks and costs of E-waste to developing countries. Indeed, E-waste is often sent for recycling and refurbishing in developing countries. The two-side proposed approach (i.e. economical and institutional) suggests that both the cost for firms to comply with increasing regulations and the imperfect implementation of regulatory restrictions lead to socially irresponsible behaviours. An increasing and better involvement of stakeholders may be put forward as a solution.

\section{INDEX}

Keywords : Corporate social responsibility, institutions, international trade, stakeholders, waste Mots-clés : Commerce international, déchets, institutions, parties prenantes, responsabilité sociale des entreprises

\section{AUTEURS}

\section{FAOUZI BENSEBAA}

Faouzi Bensebaa est docteur en sciences économiques et en sciences de gestion, habilité à diriger des recherches Ses domaines des recherches en cours portent sur la dynamique concurrentielle, la mise en œuvre de la responsabilité sociale de l'entreprise.

\section{FABIENNE BOUDIER}

Fabienne Boudier est docteur en sciences économiques, habilitée à diriger des recherches.ses domaines des recherches en cours portent sur les formes organisationnelles de la production mondialisée, les droits de propriété et responsabilité sociale des firmes multinationales. 